\title{
Hemifacial hyperhidrosis associated with ipsilateral/contralateral cervical disc herniation myelopathy. Functional considerations on how compression pattern determines the laterality
}

\author{
Satoshi Iwase, MD, PhDa \\ Yoko Inukai, MD, $\mathrm{PhD}^{\mathrm{a}}$ \\ Naoki Nishimura, $\mathrm{PhD}^{\mathrm{a}}$ \\ Maki Sato, PhDa \\ Junichi Sugenoya, MD, PhD
}

a Department of Physiology, Aichi Medical University, Nagakute, Japan

' School of Nursing, Sugiyama Jogakuen University, Nagoya, Japan

Correspondence to: Satoshi Iwase

E-mail: s_iwase@nifty.com

The authors Satoshi Iwase and Yoko Inukai contributed equally to this paper.

\section{Summary}

Sweating is an important mechanism for ensuring constant thermoregulation, but hyperhidrosis may be disturbing. We present five cases of hemifacial hyperhidrosis as a compensatory response to an/hypohidrosis caused by cervical disc herniation. All the patients complained of hemifacial hyperhidrosis, without anisocoria or blepharoptosis. Sweat function testing and thermography confirmed hyperhidrosis of hemifacial and adjacent areas. Neck MRI showed cervical disc herniation. Three of the patients had lateral compression with well-demarcated hypohidrosis below the hyperhidrosis on the same side as the cervical lesion. The rest had paramedian compression with poorly demarcated hyperhidrosis and hypohidrosis on the contralateral side. Although MRI showed no intraspinal pathological signal intensity, lateral dural compression might influence the circulation to the sudomotor pathway, and paramedian compression might influence the ipsilateral sulcal artery, which perfuses the sympathetic descending pathway and the intermediolateral nucleus. Sweat function testing and thermography should be performed to determine the focus of the hemifacial hyperhidrosis, and the myelopathy should be investigated on both sides.

KEY WORDS: cervical disc herniation, hemifacial hyperhidrosis, iodine starch method, microcirculation disorder, neck magnetic resonance imaging, thermography
Introduction

Sweating is an important mechanism for ensuring constant thermoregulation of the human body. Hyperhidrosis is defined as an excess of sweating beyond the amount required to return an elevated body temperature to normal, with a distinction drawn between primary and secondary forms. In the secondary forms, an underlying neurological or endocrinological disorder might be the cause of the profuse sweating.

Profuse sweating on the face may be disturbing and can sometimes be devastating in daily life. Although hyperhidrosis patients complain of excessive sweating, a detailed sweat function test using the two-step iodine starch method (Minor's method) in a hot environment, which demonstrates the sweating distribution, has sometimes revealed that patients have anhidrosis on the ipsi/contralateral side.

The underlying cervical lesion is usually on the side displaying anhidrosis. Minor's method involves applying an iodine solution to the skin, which is allowed to air-dry, and dusting the skin with potato starch. When sweat reaches the surface of the skin, the starch and iodine react, producing a change in color from yellow to dark blue; in this way, sweat production can be actively visualized.

Besides the Minor test (thermoregulatory qualitative sweating test), there exist thermoregulatory function evaluation techniques, such as: i) quantitative sudomotor function tests, including the ventilated capsule method and QSART (qualitative sudomotor axon reflex test), which evaluates postganlionic sudomotor function (Ogawa et al., 1975; Fealey 1997; Low and Fealey, 1999); ii) microneurography, which directly records sudomotor nerve activity (Mano et al., 2006); and iii) vasoconstriction testing upon exposure to a cold environment (Sawasaki et al., 2001). However, the advantage of the Minor test is that it clearly identifies the sweating and non-sweating areas. For this reason, we adopted this test to evaluate sudomotor function.

Early treatment with physical therapy may improve the conditions of hyperhidrosis patients. Diagnosis of these patients may be determined by sweat function tests and magnetic resonance imaging (MRI) study on the anhidrotic side. We examined five hemifacial hyperhidrosis patients, applying: i) a sweat function test using the two-step iodine starch method in a hot environment (an artificial climate chamber with ambient temperature $40^{\circ} \mathrm{C}, 50 \%$ relative humidity, $\mathrm{RH}$ ), ii) infrared thermography, where the observed sweating distribution suggests the localization of the lesion, and iii) MRI to look for cervical lesions and thus confirm the lesion. 


\section{Materials and methods}

Case 1: A 63-year-old man presented with a threemonth history of excessive right facial sweating. A year earlier, he had sustained right $9^{\text {th }}$ and $10^{\text {th }}$ rib fractures, and experienced profuse sweating on his left trunk, and subsequently sweating and hotness on the right side of his face and head. A sweat function test using the twostep iodine starch method in an artificial climate chamber demonstrated dominant sweating on the right side of the face extending to $\mathrm{C} 4$ level and on the left trunk (therefore a crossed pattern), whereas the hypohidrotic areas extended from the right $\mathrm{C} 5$ level to the abdomen, from right L5 to coccygeal level, and left T9 to coccygeal level. Thermography showed a high temperature of the skin in the areas showing hypohidrosis.

Neck MRI revealed intervertebral disc bulging, more pronounced at $3 \mathrm{~mm}$ right lateral to the midline and less pronounced at the midline of the sagittal view at C3-4 and C6-7; it compressed the right ventral side of the cervical cord slightly at C3-4 and C6-7 levels, without intramedullary signal intensity change. Low back $\mathrm{MRI}$ also demonstrated the disc herniation at L5-S1 level from the lateral side (Fig. 1). Following administration of the treatment described below, his left facial anhidrosis was slightly improved, presenting as spotlike sweating on the forehead and cheek, however, the hypohidrosis on his right trunk remained although some patchy sweating appeared.
Case 2: A 44-year-old woman presented with a threeyear history of excessive sweating and warm sensation in her face when playing tennis, regardless of the season. Examinations at an endocrinology clinic revealed no lesion in the pituitary gland. Endocrinological blood tests revealed no abnormality in luteinizing hormone, follicle stimulating hormone, prolactin, estradiol, thyroid stimulating hormone, free T3, free T4, growth hormone, or insulin-like growth factor-1 levels. Neither anisocoria nor blepharoptosis was observed. She was referred to a neurologist, and a sweat function test and thermography were performed. The sweat function test using the twostep iodine starch method in a hot environment demonstrated anhidrosis or hypohidrosis at the left face, arm, chest and abdomen. Her right face, arm, chest, and abdomen instead showed profuse sweating. Thermography showed increased skin temperature in the hypo/anhidrotic areas. MRI study revealed cervical disc herniation at $\mathrm{C6}-7$ protruding to the dural sac near the midline, but no intramedullary high intensity lesion was found (Fig. 2). As a result of the treatment described hereafter, her hemifacial anhidrosis improved, and bilateral sweating appeared, extending to her anterior chest and upper back.

Case 3: A 74-year-old woman presented with a threemonth history of profuse facial sweating, which she experienced on heat exposure and exercise. No pupillary or palpebral impairment was observed. Endo-

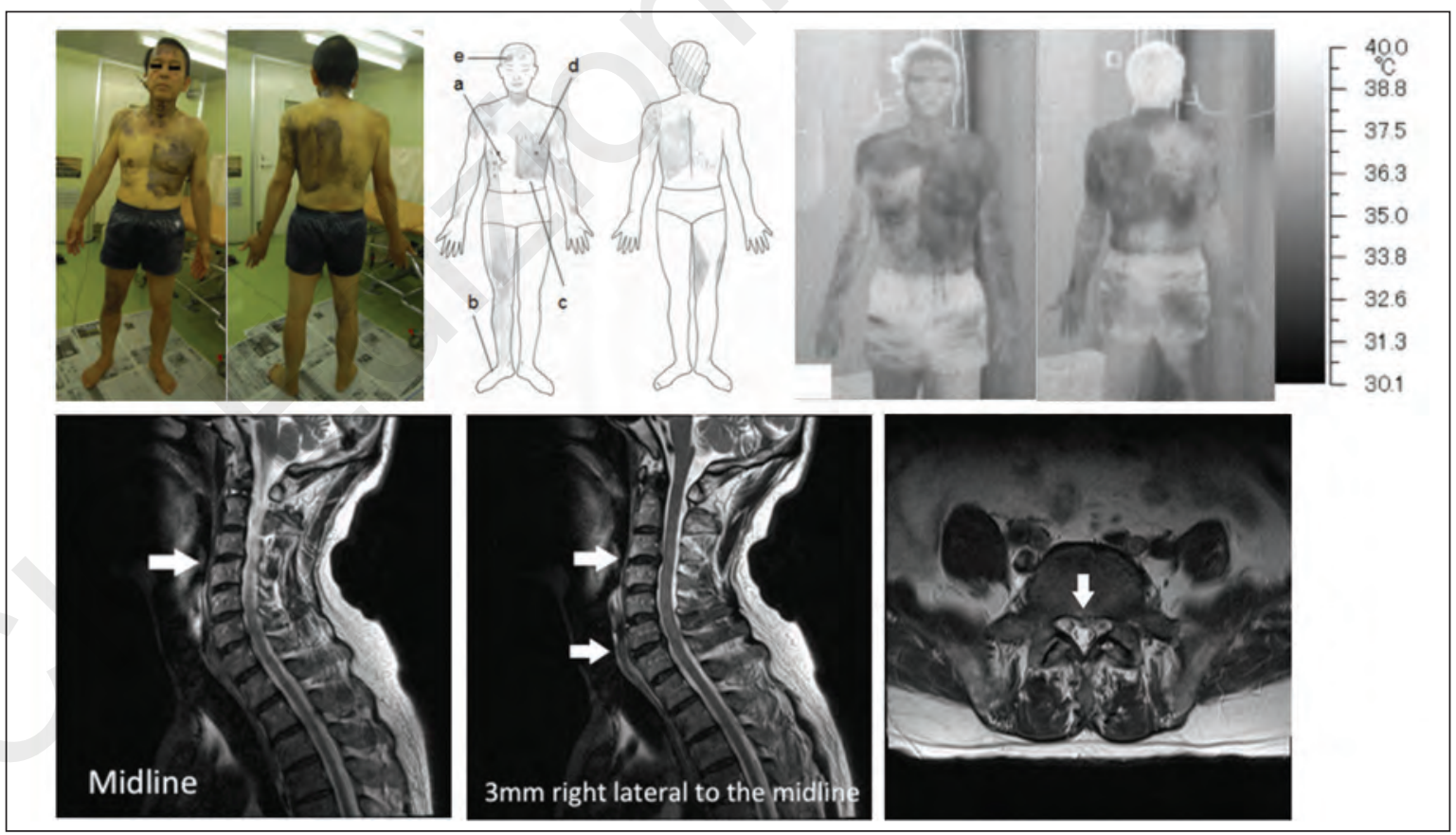

Figure 1 - Sweat distribution demonstrated by iodine starch method, thermography, and neck MRI in case 1.

The following hypohidrotic areas were observed: a) right C5 level to the abdomen, although patchy sweating, b) right L5 to the coccygeal level, c) left T9 to the coccygeal level.

The following hyperhidrotic areas were observed: d) left trunk, e) right face to C4 level.

Thermography showed a high skin temperature in the hypohidrotic area.

Neck MRI revealed intervertebral disc bulging, causing compression, more pronounced at $3 \mathrm{~mm}$ right lateral to the midline of the ventral cervical cord and only slight at the C3-4, and C6-7 level without intramedullary signal intensity change. Low back MRI showed dorsal bulging at L5-S1 level. The arrows in MRI indicate compression. 
crinological examination was normal. A sweat function test using the two-step iodine starch method in a hot environment showed anhidrosis affecting the left side of the head, face, neck and shoulder to the ring finger (C7 area), and crossed hypohidrosis at the right abdomen. Thermography showed high skin temperature in the anhidrotic and hypohidrotic areas, i.e. the left side of the face to the $\mathrm{C} 7$ area and the right side of the abdomen. Neck MRI revealed disc herniation, with bulging most pronounced at $3 \mathrm{~mm}$ right lateral to the midline of the sagittal view at the C5-6 level compressing the dural sac from the right side, although no intramedullary signal intensity change was observed (Fig. 3). Following treatment, patchy, spot-like sweat-

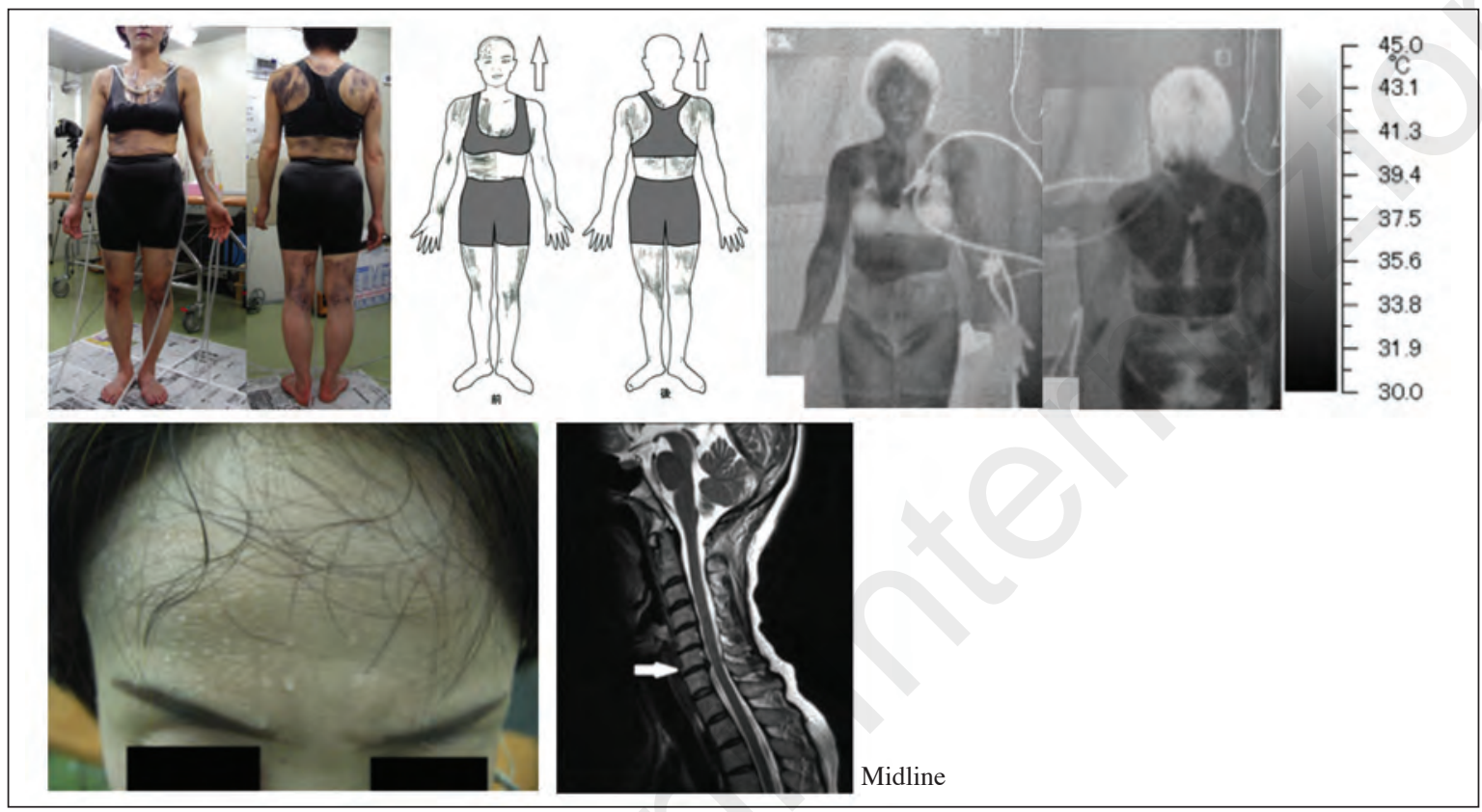

Figure 2 - Sweat distribution, thermography, and neck MRI in case 2.

Two-step iodine starch method demonstrated hypohidrosis or anhidrosis on the left face to the abdomen, and hyperhidrosis on the right face to the abdomen. (The patient refused to have iodine starch applied to her face, and we did not put the starch on her neck because of flushing caused by the starch The starch-free areas are indicated by the arrows.) Thermography showed high skin temperature in the hypo/anhidrotic areas. Neck MRI revealed intervertebral disc herniation at C6-7 level near the midline (arrow).

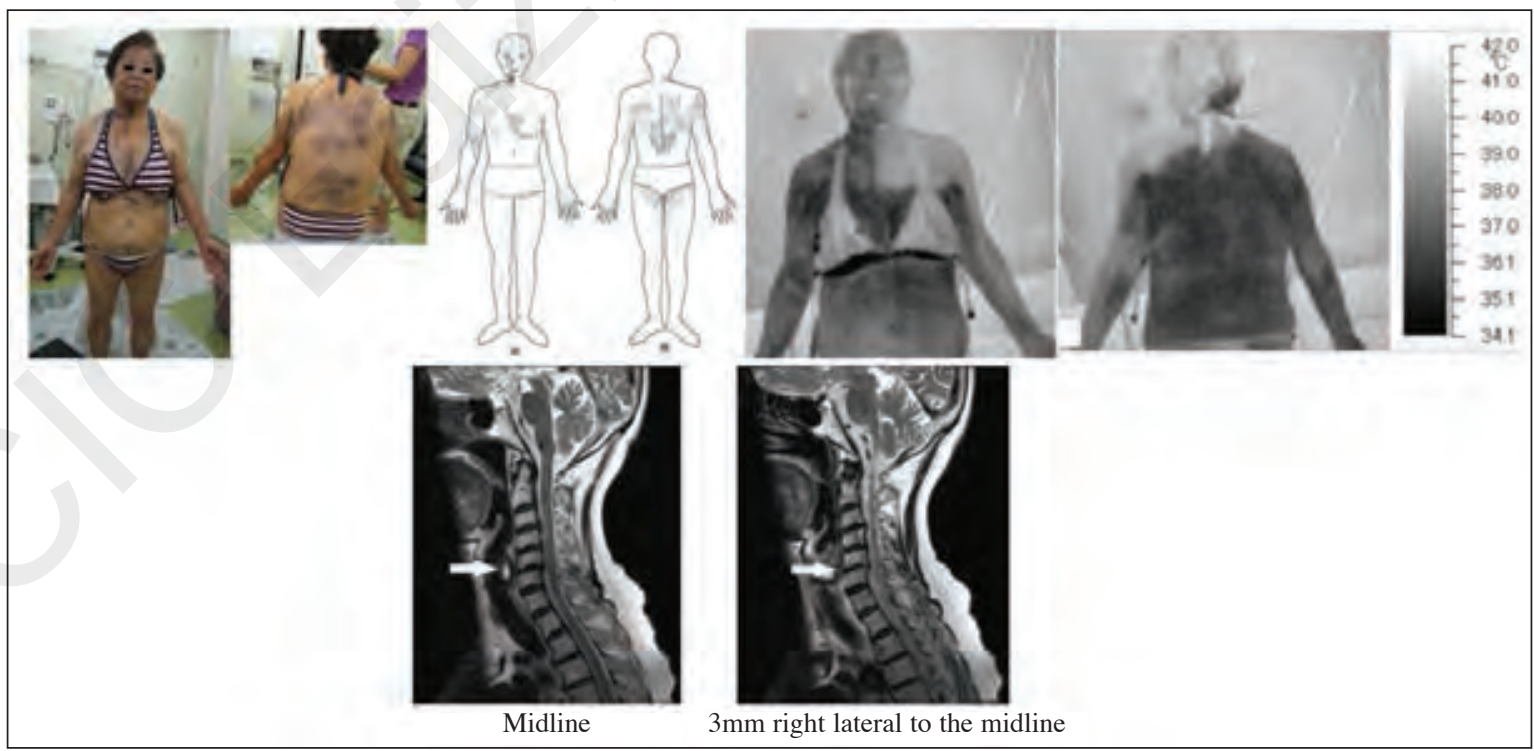

Figure 3 -Sweat distribution, thermography, and neck MRI in case 3.

Sweat function test demonstrated the anhidrosis at the left head, face, neck, shoulder to the ring finger (i.e. to $\mathrm{C} 7$ area), and crossed hypohidrosis at the right abdomen. Thermography showed high skin temperature in the anhidrotic and hypohidrotic areas, which were the left side of face to $\mathrm{C} 7$ and the right side of the abdomen. Neck MRI revealed right-sided disc herniation at the C5-6 level (arrow) compressing the dural sac. No intramedullary signal intensity change was observed. 


\section{S. Iwase et al.}

ing appeared on her left forehead, cheek and anterior neck, while her hemifacial hyperhidrosis was slightly improved. No further changes were observed in the trunk area.

Case 4: A 37-year-old woman presented with profuse sweating affecting the left side of her face and extending to the upper half of her body. The symptom had first presented two years before and gradually become marked. Two years before the onset of the hyperhidrosis, she had suffered tuberculosis, which had been completely cured with chemotherapy. Neither anisocoria nor blepharoptosis was observed. Blood chemistry and endocrinological examination were normal. A sweat function test using the two-step iodine starch method in a hot environment confirmed the profuse sweating at the left face, neck, shoulder, and arm to the C6 area of the left forearm and the palm. Left-sided anhidrosis was observed below this level (i.e. from C7 to T12), in the arm to trunk. Hypohidrosis was observed on the right side at the same level as the hyperhidrotic area, and the patient exhibited crossed anhidrosis, i.e. anhidrosis of the right face, neck, shoulder, and arm to the C6 area of the right forearm and the palm, and anhidrosis from the left C7 to T12 areas in the arm and trunk. Thermography showed high skin temperature in the anhidrotic and hypohidrotic areas. Neck MRI revealed cervical disc bulging most pronounced at $3 \mathrm{~mm}$ left lateral to the midline at C5-6 in the sagittal view, and mild compression of the dural sac from the lateral side was observed. No intramedullary signal intensity change was observed (Fig. 4). With the treatment described below, her right hemifacial anhidrosis improved, and her sweating gradually evolved into a patchy, spot-like sweating pattern. However, the anhidroisis on her trunk bilaterally has not improved in spite of the treatment.

Case 5: A 41-year-old man had suffered from massive sweating since his high school days. For the previous four months, the sweating, on exposure to the sun, exercise, or other stimulation, had been more intense on the left side of the face and chest and the right side of the dorsum manus than on other skin surfaces. Neither anisocoria nor blepharoptosis was observed. On endocrinological examination, he showed a normal thyroid function test, and normal plasma renin activity, adrenocorticotropic hormone, catecholamine fraction, aldosterone and cortisol levels. A sweat function test using the two-step iodine starch method in a hot environment showed dominant sweating in the left frontal, neck, shoulder, upper chest and low back areas. Sweating at forearms and palms was slightly more marked on the left side, but the legs showed anhidrosis. Thermography showed increased skin temperature on the right side of the whole body. Neck MRI revealed intervertebral disc herniation at C5-6, compressing the dural sac near the midline. No remarkable intramedullary signal intensity change was observed (Fig. 5). During the treatment, a carcinoid was found

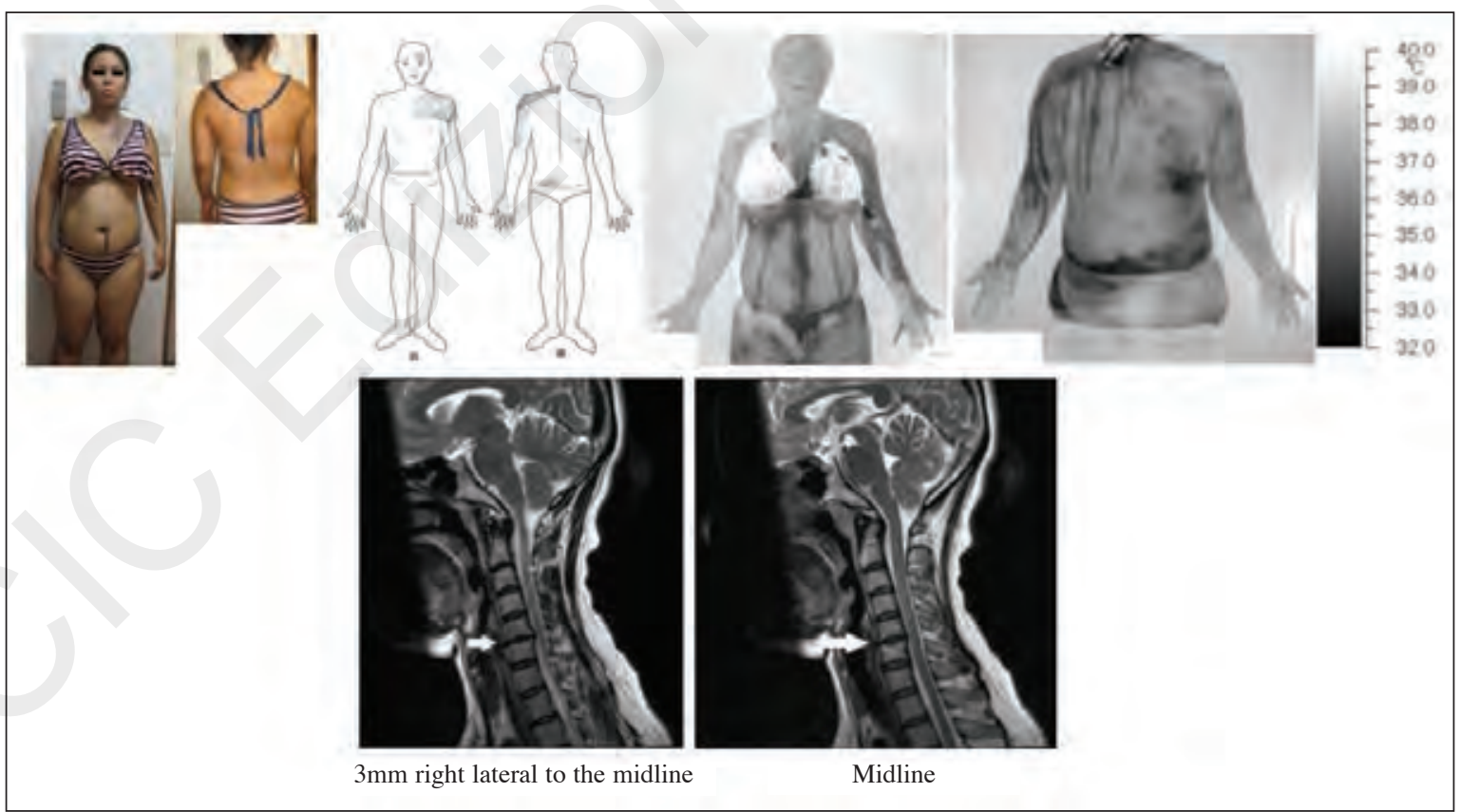

Figure 4 - Sweat distribution, thermography, and neck MRI of case 4.

Sweat function test demonstrated i) profuse sweating at the left face, neck, shoulder, and arm to the $\mathrm{C} 6$ area of the left forearm and the palm, ii) anhidrosis from the left $\mathrm{C} 7$ to T12 areas in the arm to trunk, and iii) hypohidrosis at the level of hyperhidrotic areas, but on the opposite side. The patient exhibited crossed anhidrosis, i.e. anhidrosis of the right face neck, shoulder, and arm to the $\mathrm{C} 6$ area of the right forearm and the palm, and anhidrosis from the left C7 to T12 areas in the arm to trunk. Thermography showed high temperature in the skin area corresponding to the anhidrotic and hypohidrotic areas. Neck MRI revealed the left side of cervical disc bulging at C5-6 area, and mild compression to the dural sac was observed (arrow). No intramedullary signal intensity change was observed. 


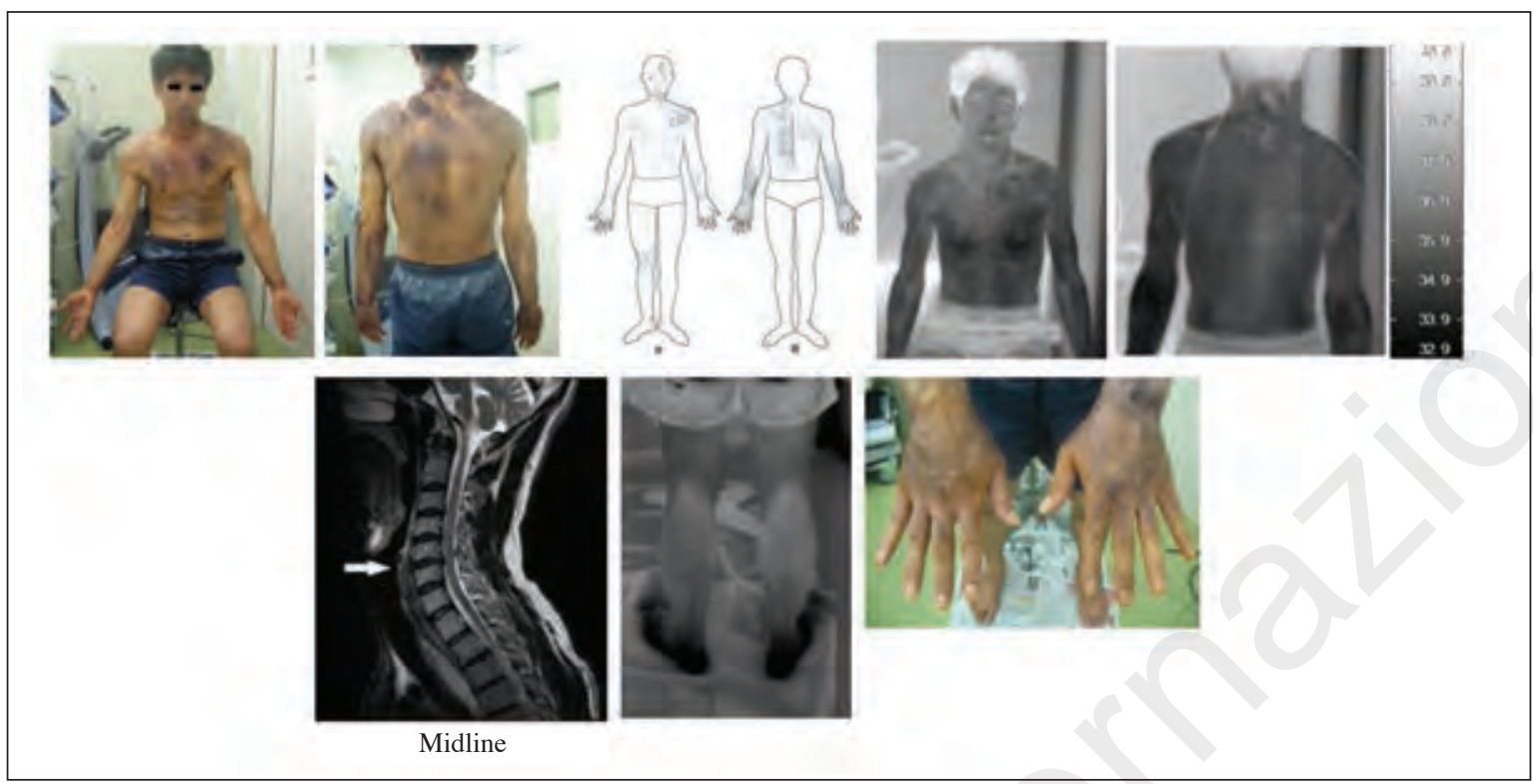

Figure 5 - Sweat distribution, thermography, and neck MRI in case 5.

Sweat function test demonstrated dominant sweating in the left frontal, neck, shoulder, upper chest and lumbar areas. Sweating at the forearms and palms was slightly more marked on the left side, but the legs showed anhidrosis. Thermography showed increased skin temperature on the right side of the whole body. Neck MRI revealed intervertebral disc herniation at C5-6 from the right side, compressing the dural sac. No intramedullary signal intensity change was observed.

near his pancreas, and removed surgically. Pathological examination revealed a catecholamine-secreting carcinoid and his hyperhidrosis ceased; asymmetrical facial sweating (right<left) persisted. Several months after the surgery, the below-described treatment was started and his facial sweating became symmetrical, with restoration of a normal sweating pattern.

\section{Treatment}

Cases 1, 2, 3 and 4 were referred for physical therapy and received neck traction, a neck collar for decompression and pharmacotherapy, consisting of methylcobalamin (vitamin B12), tocopherol nicotinate (vitamin E), and limaprost alfadex (prostaglandin E1). They obtained relief of their symptoms, and their facial hyperhidrosis was improved. Case 5 is still undergoing physical therapy.

\section{Discussion}

We described five cases presenting with hemifacial hyperhidrosis. Three of them also had hypohidrosis or anhidrosis below the hyperhidrotic area on the ipsilateral side as well as hypo- or anhidrosis at the same level as the hyperhidrosis on the contralateral side. The other two had mild hemicorporal hypohidrosis contralaterally to the hyperhidrotic area. Unlike what is seen in Horner's syndrome, they did not have anisocoria or blepharoptosis; furthermore, they did not complain of facial flushing, which thus distinguished their condition from harlequin syndrome (Lance et al., 1988).
The sweating distribution observed in case 3 suggested that the localization might be at the left cervical sympathetic trunk, or the right sympathetic descending nerve in the cervical spinal cord, and the MRI study revealed right-sided C5-6 disc herniation. This finding is compatible with the level of the upper margin of the patient's right hypohidrotic area because the compression level and anhidrotic area have a gap of a few levels. Therefore the cervical disc herniation might also have been involved in the hypohidrosis in the right abdominal area. We conclude that the hypohidrosis on her right lower trunk was caused by the rightsided disc herniation. The sweating distribution of case 4 suggested a focal localization in the right cervical sympathetic trunk or the left sympathetic descending tract in the cervical spinal cord, and the MRI study revealed cervical disc bulging on the left side at C5-6 level. This suggests that this left-sided cervical disc bulging was the lesion responsible for the left anhidrosis she displayed below the $\mathrm{C} 7$ area. In cases 1, 3 and 4, the level of the upper margin of their well-demarcated anhidrosis corresponded to the level of their cervical disc bulging. Furthermore, their an/hypohidrotic side was ipsilateral to their disc bulging side. However, since the intermediolateral nucleus does not exist at the level of the cervical spinal cord (it exists only at the levels T1 to L2/3), the relationship between anhidrosis and level of cervical spinal involvement remains to be clarified (Jansen and Loery, 1997).

By contrast, the sweating distribution shown by case 2 , characterized by anhidrosis or hypohidrosis extending from the left side of the face to the abdomen, suggested that the lesion might be in the left sympathetic 
descending tract from the hypothalamus to the cervical spinal cord, and the MRI study revealed a cervical disc herniation at 66-7 near the midline. In case 5, the results of the sweat function test, which showed hypohidrosis extending from the right side of the face to the abdomen, suggested a lesion in the right sympathetic descending tract from the hypothalamus to the cervical spinal cord, and the MRI revealed herniation of the intervertebral disc at C5-6 near the midline. The levels of disc herniation were compatible with the an/hypohidrotic distribution in these two cases, while the mechanism underlying the laterality of their sweating is not clarified by their disc herniation near the midline. All the patients underwent physical therapy (neck traction, neck collar for decompression, etc.), even though their MRI examinations showed only dural sac compression, and no intramedullary signal intensity change, and the conditions of four of them improved. No patient showed motor or sensory neuropathy or somatic myelopathy on neurological examination.

Neuroanatomically, the first neuron of the sympathetic fibers begins in the posterior hypothalamus, traverses the midbrain and reticular formation of the pons, and ends in an intermediolateral nucleus in the spinal cord. The second neuron begins with the white rami communicantes of $\mathrm{C} 8$ to T2, ascends the stellate ganglion and sympathetic trunk to synapse at the superior cervical ganglion, where it completes its course (Walsh, 1992). Lesions along this pathway can cause Horner's syndrome or harlequin syndrome (Lance et al., 1988; Drummond and Lance, 1993).

Our cases presented only hemifacial hyperhidrosis and had no facial flushing, anisocoria or blepharoptosis; minor compression of the cervical spinal cord by disc herniation was observed in all cases without intramedullary signal abnormalities on MRI. This suggests that, despite the absence of abnormal signals in the spinal cord, the premotor neuron from the hypothalamus to the intermediolateral nucleus was impaired by the disc herniation. Since the disc herniation myelopathy was observed on the side ipsilateral to the anhidrotic or hypohidrotic area, we assume this anhidrosis or hypohidrosis can be attributed to the disc herniation myelopathy.

Our five cases showed two patterns of compression by disc herniation: lateral and paramedian. The lateral compression pattern was found in cases 1, 3 and 4, and the paramedian pattern in cases 2 and 5 . In the patients showing lateral compression, the anhidrotic or hypohidrotic area was clearly demarcated by the hyperhidrotic and hypohidrotic areas corresponding to the spinal levels on the bulging side. By contrast, the paramedian compression cases exhibited mild hypohidrosis in vaguely demarcated hemicorporal areas. It is not known why lateral compression produces clear demarcation whereas paramedian compression induces generalized vague demarcation of the anhidrotic or hypohidrotic areas, however, it is likely that blood supply patterns in the spinal cord play a role.

In the lateral compression cases $(1,3$, and 4$)$, the level of the upper margin of the well-demarcated anhidrotic areas corresponded to the level of compression by the disc herniation, which indicates that the first sudomotor neuron of sympathetic fibers may be influenced by such lateral bulging. Nathan and Smith (1986) reported that anterolateral cordotomy of the cervical spinal cord revealed that most of the fibers supplying the sudomotor neurons lay near the posterior angle of the anterior horn, although some lay outside this area. The mechanism underlying the anhidrosis present below the level corresponding to the compression site at cervical level in the lateral (or, more precisely, "anterolateral type") compression cases also needs to be explained. It is possible that the somatotropic representation of the sudomotor sympathetic tract is laminarly organized from the lateral side in the following sequence: sacral, lumbar, thoracic, and cervical, as observed in the lateral corticospinal tract. When the intervertebral disc bulges anterolaterally, the premotor neurons of the sympathetic sudomotor tract are naturally the ones most influenced by this bulging since they descend just lateral to the posterior angle of the anterior horn (Nathan and Smith, 1986). The ipsilateral areas above the areas of anhidrosis caused by the bulging disc exhibited vertical compensatory hyperhidrosis, as is often observed after the endoscopic thoracic sympathectomy (Chwajol et al., 2009; Cerfolio et al., 2011). The hypohidrosis or anhidrosis exhibited in the contralateral area at the same level as the hyperhidrosis (resulting in a crossed hypohidrotic or anhidrotic area below the lesion) might constitute relative hypohidrosis compensatorily reacting to the contralateral hyperhidrosis. In the cases (2 and 5 ) showing paramedian compression near the midline, the sudomotor pathway from the hypothalamus to the intermediolateral cell column might be impaired through reduction in the vascular supply of the ipsilateral sulcal artery arising from the anterior spinal artery, which has been shown to cause hemicorporal poorly demarcated hypohidrosis on the ipsilateral side (Erra et al., 1991). Smith and Smith (1999) reported that one patient with bilateral Horner's syndrome had bilateral interruption of the cervical sympathetic pathway caused by an anterior spinal artery thrombosis at the level of C5-6. li et al. (2009) reported that elevation of venous pressure in the craniospinal axis and intervertebral disc pressure might cause minor damage and intraluminal thrombus in the anterior spinal artery. These patterns indicate that even a minor circulatory insufficiency at the cervical cord might cause impairment of the vulnerable sudomotor sympathetic descending pathway, however, these lesions did not appear as abnormal signals on MRI. The symptoms were improved by the above-described treatment, which showed that the hemilateral hyperhidrosis was due to the spinal lesion, specifically disc hernia. Although the carcinoid possibly contributed to the hyperhidrosis in case 5, asymmetrical facial sweating was relieved by the treatment.

The literature contains reports of tumor-related pathogenesis in hemifacial hyperhidrosis, i.e., bronchial carcinoma (Poh, 1978), intrathoracic neoplasia (Lindsay et al., 1986), pancoast tumor (Yamauchi et 
al. 1994), and spinal cord tumor (Kilinçer et al., 2007). Therefore, the patients were thoroughly investigated for intrathoracic lesions, but none was found in any of the cases. Another possible explanation for the segmental hyperhidrosis was neuropathy associated with Sjögren's syndrome (Kumazawa et al., 1993), however, none of the patients presented symptoms related to Sjögren's syndrome, either glandular ones, including dry eyes, dry oral cavity, dysphagia, or extraglandular ones, including fatigue, joint pain, arthritis, or other inflammatory findings.

Another possibility to consider in hemifacial hyperhidrosis is involvement of sensory input. Indeed, disturbance of sensory feedback to the neck region might be a possible cause of hemifacial hyperhidrosis. Gorman (2010) reported unilateral hyperhidrosis in spinal cord injury with $\mathrm{C} 4$ complete tetraplegia. The left hemifacial hyperhidrosis was aggravated when pressure was applied to the right ischium for several minutes, but then subsided on release of the pressure. Subdermal injection of lidocaine and application of an air cushion on the right ischium relieved the condition, which suggested that the nociceptive irritation of sensory input might have been the cause. Yoshimura et al. (2012) reported a case of gustatory sweating in the submandibular region after selective neck dissection surgery due to aberrant nerve regeneration. However, our five cases presented no specific sensory disturbance in the course of hemifacial hyperhidrosis.

In conclusion, lateral minor compression on the cervical spinal cord may cause ipsilateral well-demarcated anhidrosis below the compressed level, and simultaneously ipsilateral compensatory hyperhidrosis above the anhidrotic area. Sometimes crossed hypohidrosis may be observed on the contralateral area at the same level as the hyperhidrosis. Compression near the midline (paramedian compression) may cause generalized hemicorporal poorly-demarcated anhidrosis or hypohidrosis, and simultaneously compensatory hyperhidosis on the contralateral face and upper trunk. In patients with hemifacial hyperhidrosis, sweat function testing using the two-step iodine starch method should be performed whenever possible to determine the anhidrotic and hyperhidrotic areas, as well as thermography to determine the localization of the cervical lesion, i.e. the side and the level; and spinal lesions should be investigated on the side ipsi/contralateral to the hyperhidrosis.

\section{References}

Arai $\mathrm{S}$, Utsunomiya $\mathrm{H}$, Wakugawa $\mathrm{Y}$, et al (2007). A case of spinal cord infarction caused by cervical disc herniation. Brain Nerve 59: 997-1000.

Cerfolio RJ, De Campos JR, Bryant AS, et al (2011). The Society of Thoracic Surgeons expert consensus for the surgical treatment of hyperhidrosis. Ann Thorac Surg 91: 1642-1648.

Chwajol M, Barrenechea IJ, Chakraborty S, et al (2009). Impact of compensatory hyperhidrosis on patient satisfaction after endoscopic thoracic sympathectomy. Neurosurgery 64: 511-518.

Drummond PD, Lance JW (1993). Site of autonomic deficit in harlequin syndrome: local autonomic failure affecting the arm and the face. Ann Neurol 34: 814-819.

Erra JM, Ara JR, Pina MA, et al (1991). Anterior spinal artery syndrome caused by cervical disc protrusion. Diagnosis by magnetic resonance. Neurologia 6: 256-258.

Fealey RD (1997) Thermoregulatory sweat test. In: Low PA (Ed.) Clinical Autonomic Disorders, 2nd ed. Philadelphia, Lippincott-Raven, pp. 245-257.

Gorman PH (2010). Unilateral hyperhidrosis from a contralateral source in an individual with C4 complete tetraplegia. $J$ Spinal Cord Med 33: 428-430.

li Y, Maki T, Furuta T, et al (2009). Cervical spinal cord infarction in a patient with cervical spondylosis triggered by straining during bowel movement. J Clin Neurosci 16: 106-107.

Jansen AS Loewy AD (1997). Neurons lying in the white matter of the upper cervical spinal cord project to the intermediolateral cell column. Neuroscience 77: 889-898.

Kilinçer C, Oztürk L, Hamamcioglu MK, et al (2007). An upper thoracic spinal cord tumor presenting as hemifacial hyperhidrosis. Surg Neurol 68: 461-463.

Kumazawa K, Sobue G, Yamamoto K, et al (1993). Segmental anhidrosis in the spinal dermatomes in Sjögren's syndrome-associated neuropathy. Neurology 43: 1820-1823.

Lance JW, Drummond PD, Gandevia SC, et al (1988). Harlequin syndrome: the sudden onset of unilateral flushing and sweating. J Neurol Neurosurg Psychiatry 51: 635-642.

Lindsay DC, Freeman JG, Record CO (1986). Unilateral hyperhidrosis associated with underlying intrathoracic neoplasia. Thorax 41: 814-815.

Low PA, Fealey RD (1999) Evaluation of sudomotor function. In: Mathias CJ, Bannister Sir R (Eds) Autonomic Failure. A Textbook of Clinical Disorders of the Autonomic Nervous System, 4th ed, Oxford University Press, Oxford, pp. 263270.

Mano T, Iwase S, Toma S (2006) Microneurography as a tool in clinical neurophysiology to investigate peripheral neural traffic in humans. Clin Neurophysiol 117: 2357-2384.

Nathan PW, Smith MC (1986). The location of descending fibres to sympathetic neurons supplying the eye and sudomotor neurons supplying the head and neck. J Neurol Neurosurg Psychiatry 49: 187-194.

Ogawa T (1975) Thermal influence on palmar sweating and mental influence on generalized sweating in man. Jpn J Physiol 25: 525-536.

Poh SC (1978). Bronchial carcinoma with hemilateral hyperhidrosis. Singapore Med J 19: 59-60.

Sawasaki N, Iwase S, Mano T (2001) Effect of skin sympathetic response to local or systemic cold exposure on thermoregulatory functions in humans. Auton Neurosci 87: 274-281.

Smith SA, Smith SE (1999). Bilateral Horner's syndrome: detection and occurrence. J Neurol Neurosurg Psychiatry 66:48-51.

Yamauchi Y, Kobayashi T, Nagano T, et al (1994). A case of hemifacial hyperhidrosis on the opposite side of the pancoast tumor. Masui 43: 924-926.

Yoshimura H, Tobita T, Kumakiri M, et al. (2012). Gustatory sweating in the submandibular region following neck dissection: a case with thermographic evaluation and review of the literature. J Oral Maxillofac Surg 70:e667-673.

Walsh TJ (1992). Pupillary abnormalities. In: Walsh TJ (Ed.), Neuro-Ophthalmology Clinical Signs and Symptoms, Philadelphia, Pennsylvania, Lea \& Febiger, pp. 62-75. 